\title{
Forensic Investigation of a Breakdown Waste Water Pipeline off Penghu Islands, Taiwan \\ Wen-Miin TIAN
}

\author{
National Sun Yet-sen University, 70, Lien-Hai Road, Ku-Shan District, Kaohsiung City, Taiwan 804
}

tiwemi@mail.nsysu.edu.tw

Keywords: Offshore Pipeline, Side-scan Sonar, Image Resolution, Target Detection.

\begin{abstract}
The causes and mechanism inducing the breakdown and follow up displacement of an outflow pipeline off Penghu Islands, Taiwan was investigated. Based on the information illustrated in the high resolution side-scan sonar imagery, it was concluded that the breakdown of the pipeline was initiated by a large external pulling force. In addition, this pulling force dragged the breakdown pipeline westward with a net displacement of about $100 \mathrm{~m}$. After the pulling force disappeared, environmental forces such as tidal currents interacted with the floating section of the breakdown pipeline caused a further and complex displacement pattern of the breakdown pipeline especially in the portion near the outlet. At the moment of this forensic investigation, the overall appearance of the breakdown pipeline illustrated an irregularly-shaped circle. Further displacement of the breakdown pipeline in accordance with sea states is highly expected.
\end{abstract}

\section{Introduction}

The offshore saline water and/or waste water outflow pipeline of Hsiyu Seawater Desalination Plant, located at Penghu County, Taiwan, was deployed and completed in June, 2008. The total offshore length of this HDPE (high density polyethylene) pipeline is 3,000 m with outer diameter of $315 \mathrm{~mm}$. Water depth along this pipeline varied from coast line to a maximum of $45 \mathrm{~m}$ at the offshore outlet point and the seabed substrate consists mainly sediment of medium sand. For the purpose of improving stability of the offshore pipeline system on the seafloor, the pipeline was wrapped with concrete loading blocks of $20 \mathrm{~kg}$ in weight at each $2 \mathrm{~m}$ interval. After the deployment, a proof inspection was conducted and reported that the pipeline was installed properly as designed. However, in September, 2008, a mandatory and regularly conducted underwater video inspection along the pipeline by divers indicated that the pipeline was breakdown at the location with water depth of $36 \mathrm{~m}$. Evidences left on the breakage area illustrated that the pipeline was under extensive pulling activities by massive and extended external force before breakdown. The breakdown pipeline from the breakdown point to the outlet point was estimated to be $500 \mathrm{~m}$ in length and was missing even though the divers conducted several comprehensive searching for operations around that area thereafter.

For the purpose of locating and mapping this $500 \mathrm{~m}$ breakdown and missing pipeline, collecting detailed information regarding the status of this pipeline, and deriving causes and mechanism which induced the breakdown of this pipeline, a forensic investigation was granted and conducted in December, 2008, and was concluded and reported in January, 2009. This manuscript was dedicated in the description of the methodology and survey strategy employed in this investigation for underwater in-situ information collection, status of the detected pipeline derived from manual observation of the collected information, and conclusions regarding the initiation and mechanism causing the breakdown and the subsequent missing state of the pipeline.

\section{Methodology and Survey Strategy}

For the purpose of searching for and collecting detailed information regarding the missing and proud HDPE pipeline with concrete loading blocks, a $500 \mathrm{kHz}$ side-scan sonar system with an integrated Global 
Positioning System (GPS) and digital nautical charts for real-time survey guidance was proposed and employed in this investigation [1].

A side-scan sonar produces images of the seafloor, made of points (pixels) whose values are proportional to the amount of energy backscattered, which are expressed as grey levels. The backscattering is affected, in decreasing importance, by the geometry of the sensor-target system (relative angle of ensonification), the morphological characteristics of the surface (e.g., micro-scale roughness) and by its intrinsic nature (composition, density, impedance, relative importance of volume and surface reverberation) [2, 3, 4]. Acoustic impedance (the product of density, and compressional wave velocity, v) can be used to define the reflection coefficient, $\mathrm{R}$ for normal incidence, which is a measure of the strength of the reflection [5]:

$$
R=\frac{\rho_{2} \cdot v_{2}-\rho_{1} \cdot v_{1}}{\rho_{2} \cdot v_{2}+\rho_{1} \cdot v_{1}}
$$

For a HDPE pipeline and accompanied concrete loading blocks, the reflection coefficient (R) for normal incidence with respect to sea water is approximately 0.46 . The reflection coefficient of typical sandy sediments is approximately 0.33 [6]. Therefore, a proud pipeline lying on sandy sediment will acoustically appear as slightly dark and linear scatters accompanied by shadows. The accompanying shadow, if any, will depend on the height of the pipeline and concrete blocks extended above the seafloor and the altitude of the sonar towfish above the bottom [3, 4]. In order to get a comprehensive understanding of the pipeline location in the investigated area, the geocoded side-scan sonar image was further processed, which included: geometric corrections (i.e., slant range to ground range), radiometric corrections (i.e., time-varying gain corrections) and mosaicking.

A survey strategy which included two interdependent stages were adopted and planned in this investigation. An initial field survey in a broad scale was dedicated for the detection and verification of the missing pipeline. After the completion of the first survey stage and the missing pipeline was detected and positioned, a detailed survey for the collection of comprehensive pipeline information at various aspects was conducted. At the first stage, the planned survey was intended to cover an area of at least 1 square nautical mile with the pipeline breakout point and the deployed outlet point included inside the survey area. Systematic parallel, north-south bound (i.e., latitudinal), and equally spaced survey routes were assigned. At the second stage, a repetition surveying strategy with survey routes across the pipeline and higher sonar image resolution settings (i.e., shorter range settings) was employed for the collection of detailed information regarding the breakout pipeline. Based on the high resolution side-scan sonar imagery, survey routes, and the production of georeferenced mosaics, detailed information regarding the status, geographic coordinates, and prominent features of the breakdown pipeline were established for further evaluation of the initiation and mechanism of the breakdown of the pipeline.

\section{Results}

Due to continuous rough sea states during winter time around Taiwan offshore area, it is a challenge to the surveying operators to collect effective information for the detection and identification of the missing breakdown pipeline. Under this circumstance, a revised and straightforward version of the survey operation was managed which incorporated the first and second surveying stages together and collect all the necessary information within four hours at sea.

The breakdown pipeline, with total length of $500 \mathrm{~m}$ and overall appearance of an irregularly-shaped circle, was located at a minimum distance of $50 \mathrm{~m}$ to the north-western direction of the initially recorded breakdown point (Fig. 1 \& Fig.2). Both ends of the breakout pipeline (i.e., breakout point and outlet) were located at the north-western corner of the circle area. Concrete loading blocks were intact and regularly distributed along the pipeline.

Two prominent features were detected along the breakout pipeline which included a double twisted area with extraordinary sharp angle (Fig. 3) and a section of pipeline floated off the seafloor (Fig. 4 \& Fig. 
5). The double twisted pipeline area is located close to the breakdown point and the straight line distance in between is about $70 \mathrm{~m}$. On the other hand, the floating pipeline area is located close to the outlet and the minimum straight line distance in between is about $80 \mathrm{~m}$. The section of the floated pipeline, where at least 10 evenly spaced concrete loading blocks were identified, extended approximately $30 \mathrm{~m}$ horizontally and the maximum height off the seafloor is about $8.5 \mathrm{~m}$.

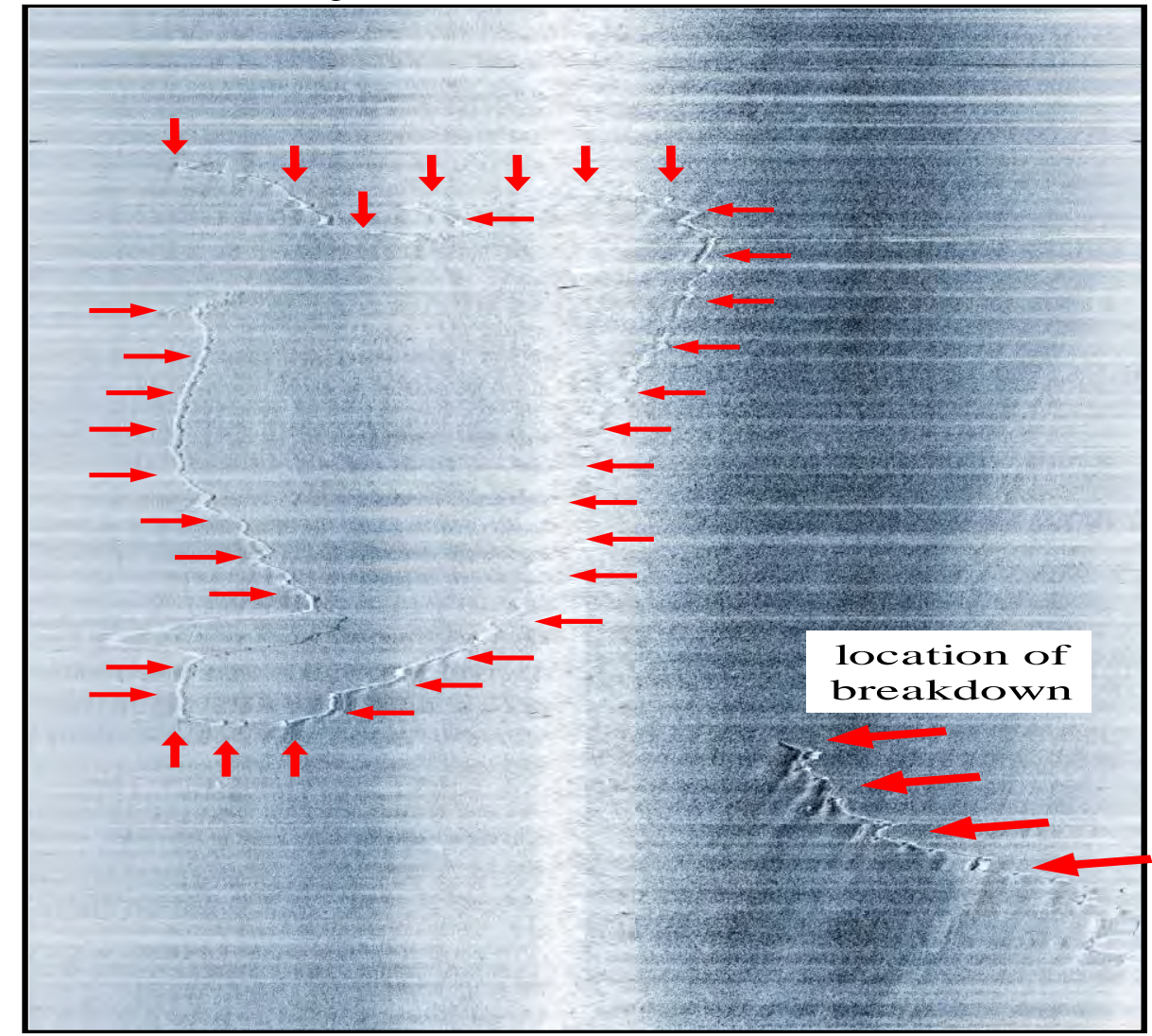

Fig. 1 Side-scan Sonar Imagery of the Breakdown Pipeline which Illustrated an Irregularly-shaped Circle.Red Arrow Indicates the Location of the Concrete Loading Block

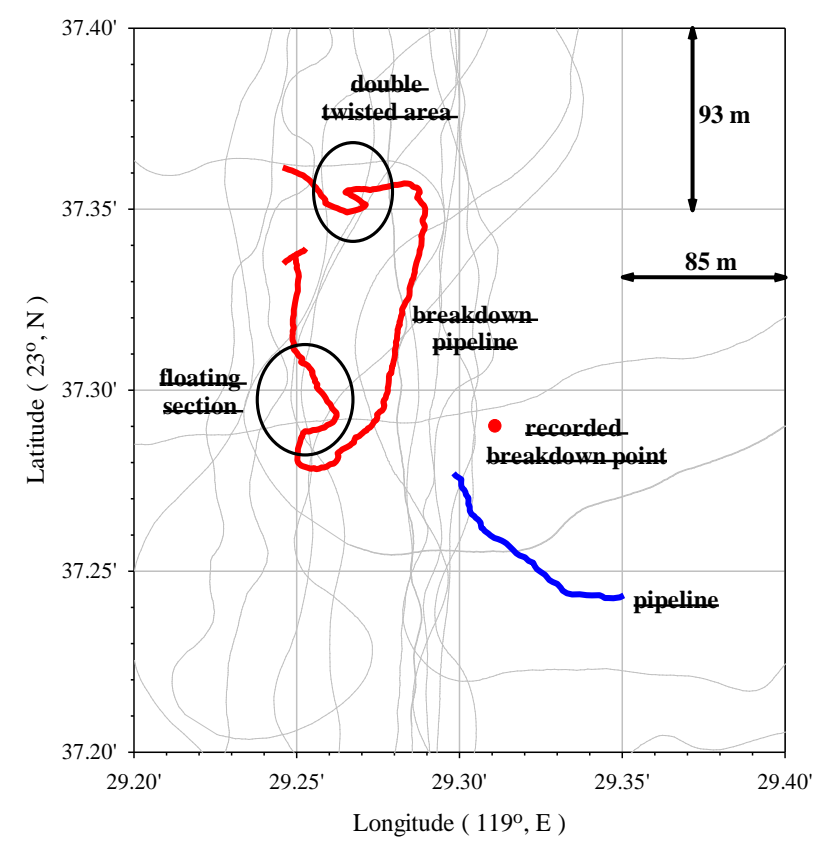

Fig. 2 Geographic Location and Overall Appearance of the Breakdown Pipeline

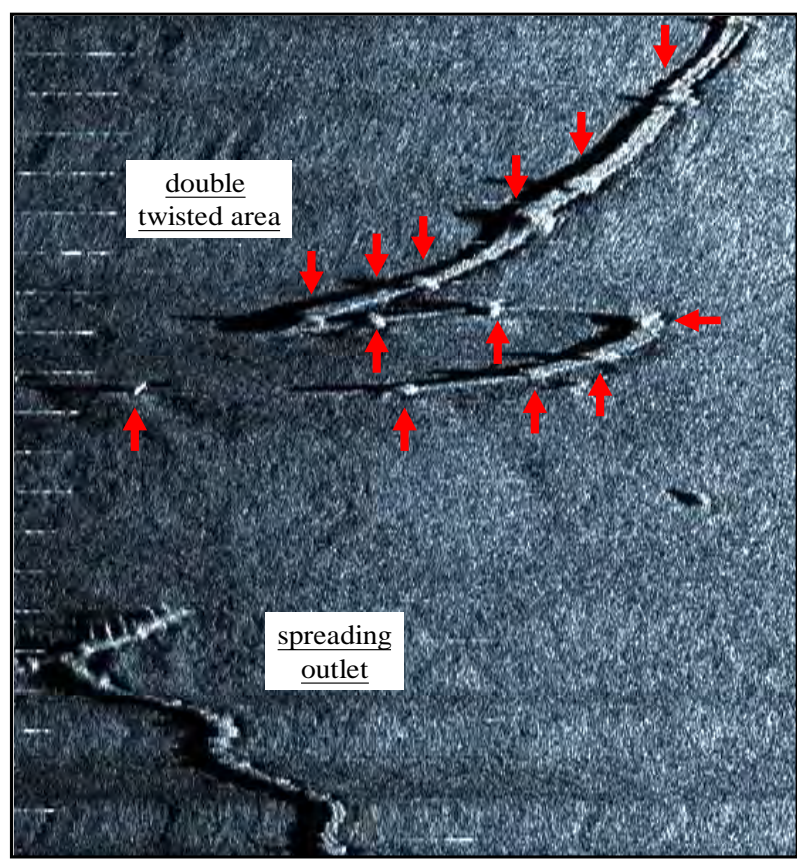

Fig. 3 Double Twisted Pipeline Area with Sharp Angle 


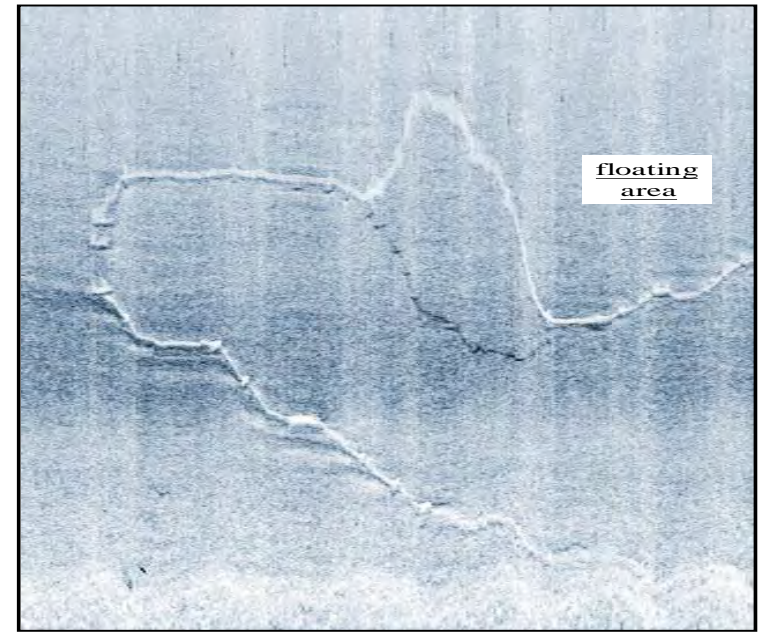

Fig. 4 Section of the Pipeline Floated off the Seafloor-1

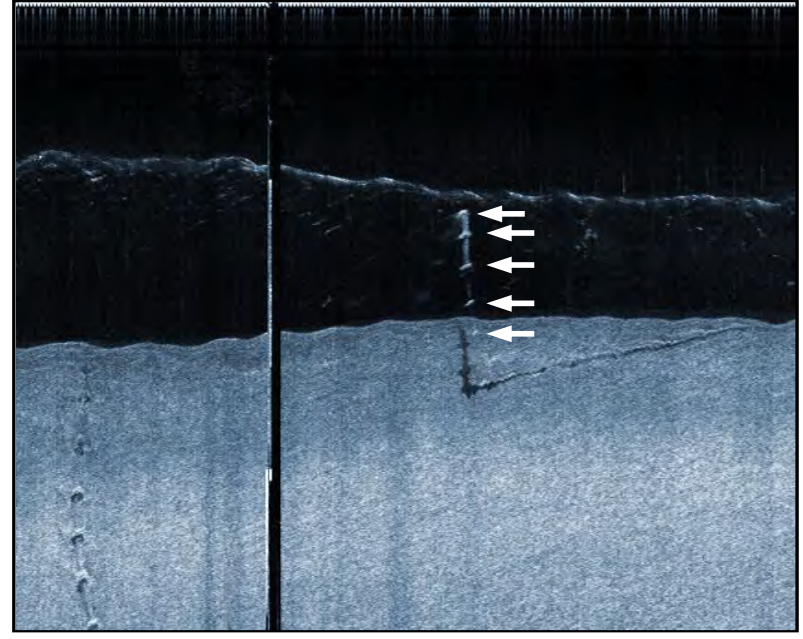

Fig. 5 Section of the pipeline floated off the Seafloor-2

\section{Conclusion}

Based on a mutual consideration of the pipeline related documents such as pipeline deployment records and optical inspection records as well as environmental influencing factors such as sea states and fisherman activities around this area, it was concluded that breakdown of the pipeline was initiated by a large external pulling force. In addition, this pulling force dragged the breakdown pipeline to the west with a net displacement of about $100 \mathrm{~m}$. After the pulling force disappeared, environmental forces such as tidal currents interacted with the floating section of the breakdown pipeline caused a further and complex displacement pattern of the breakdown pipeline especially in the portion near the outlet. At the moment when this forensic investigation was conducted, the overall appearance of the breakdown pipeline illustrated an irregularly-shaped circle. It should be emphasized that further displacement of the breakdown pipeline in accordance with sea states is highly expected.

\section{Acknowledgement}

This investigation was financially supported by KUO TOONG Internal Co. LTD.

\section{References}

[1]W. M. Tian, W. M. (2008), Integrated method for the detection and location of underwater pipelines, Applied Acoustics, 69(5), (2008) 387-398.

[2]Ph. Blondel, B. J. Murton, Handbook of Seafloor Sonar Imagery, Praxis Publishing Ltd, 1997.

[3] $\mathrm{Ph}$. Blondel, Automatic mine detection by textural analysis of COTS sidescan sonar imagery, International Journal of Remote Sensing, 21(6), (2000), 3115-3128.

[4]X. Lurton, An Introduction to Underwater Acoustics: Principles and Applications, Praxis Publishing Ltd, 2002.

[5]L. A. Kinsler, A. R. Frey, A. B. Coppens, J. V. Sanders, Fundamentals of Acoustics, John Wiley \& Sons, Inc., 1982.

[6]M. P. Chen, Y. C. Chern, Sedimentary Structure and Acoustic Characteristics of Surficial Sediments off Southwest Taiwan, National Taiwan University, 1997. 